\title{
"Mastering" the Laboratory Sciences in Public Health Education
}

\author{
Joshua Geltz, M.S., Ph.D. ${ }^{1,2}$, Prezley Duncan, M.S. ${ }^{1}$, E. Matt Charles B.S. ${ }^{2}$, Robert Cox, MPH, MT(ASCP)ㄹ, Andrea Stadsholt, B.S., \\ MT(ASCP) ${ }^{2}$, David Carpenter, Ph.D. ${ }^{1}$, Donald S. Torry, Ph.D. ${ }^{1,3}$, Andrew Wilber, Ph.D. ${ }^{1,3 *}$ \\ ${ }^{1}$ Department of Medical Microbiology, Immunology and Cell Biology, Southern Illinois University School of Medicine, Springfield, IL 62702 \\ ${ }^{2}$ Illinois Department of Public Health, Division of Laboratories, Springfield, IL 62702 \\ ${ }^{3}$ Simmons Cancer Institute, Springfield, IL 62702
}

${ }^{\star}$ Corresponding author: Andrew Wilber, Department of Medical Microbiology, Immunology and Cell Biology, Southern Illinois University School of Medicine, 825 North Rutledge, Room 2633, Springfield, IL 62702; E-mail: awilber@siumed.edu

Received: April 29, 2020; Accepted: May 05, 2020; Published: May 12, 2020

\begin{abstract}
Public health laboratories provide critical surveillance data to improve community health and environmental quality. Yet, the nation is facing a large shortfall in laboratory workers and leaders due to the number of those projected to retire and the increasing complexity of analyses and regulatory requirements. To help meet this demand, the Public Health Laboratory Sciences Master's degree program at Southern Illinois University School of Medicine combines graduate-level classroom instruction with extensive research experience in a public health laboratory. Developed as a collaborative effort with the Illinois Department of Public Health, this graduate program is designed to produce scientists with applied training in clinical, environmental, and molecular testing protocols. The diverse training environment allows for immediate employment of graduates who require only minimal additional "on the job" training. This article will highlight the significance, curriculum, training, and success of this unique master's degree dedicated to training the next generation of public health laboratory employees and leaders.
\end{abstract}

Keywords: Public Health, Laboratory, Education

\section{Introduction}

As the U.S. population continues to expand, so too has the demand for a highly-skilled public health workforce. However, the public health community faces current and future challenges to ensure that public health services are sustained for future generations. Many of these challenges have been discussed in length by the American Public Health Association (APHA) and include: i) budget cuts and lack of appropriate funds to support public health services, ii) significant reduction in the public health workforce (up to $50 \%$ was expected by 2012) due to retirement, iii) decrease in well qualified entry-level public health professionals to replace retirees and iv) increasing demand for more technical and analytical experts for current and future testing methodologies [1]. Despite a shrinking budget and workforce, public health agencies have been challenged to provide the same level and quality of services. The ideal ratio of public health professionals is based on the 1980 model of 220 public health professionals for every 100000 U.S. citizens [2]. While the U.S. population has increased $27 \%$ since 1980 , the public health workforce has decreased by around 55000 to reflect today's working capacity of $\sim 500000$ employees [3-5]. In order to achieve the appropriate number of public health professionals for current U.S populations, the workforce would need to add $\sim 750000$ public health professionals over the next 8 to 10 years. To address this demand, Southern Illinois University School of Medicine (SIU-SOM) in collaboration with the
Illinois Department of Public Health (IDPH) developed a unique academic and laboratory training program to reinforce the ranks of the public health workforce.

\section{Present and future deficiencies of the public health workforce}

As defined by the Institute of Medicine, a public health professional is "a person educated in public health or a related discipline who is employed to improve health through a population focus" [6]. Of the collective public health professionals about 16000 (3.1\%) are laboratorians. However, only $20 \%$ of individuals received formal training within their assigned laboratory occupation [7]. In addition, the number of personnel with advanced graduate degrees needed to successfully implement, conduct, and troubleshoot protocols performed within a modern laboratory are inadequate with only $33 \%$ of laboratorians holding a Master of Public Health (MPH) or equivalent advanced degree [8]. Classic examples outlining the responsibilities of properly educated and trained public health laboratory professionals include the identification of infectious pathogens during epidemic and pandemic outbreaks, newborn screening for heritable genetic diseases, environmental testing in support of the Safe Drinking Water Act, and screening of clinical samples to identify sexually transmitted diseases. In addition to an increasing U.S. population, modern medicine and preventative measures continue to decrease morbidity and mortality of acute and chronic disease which increases life expectancy. Despite advancements in the quality and longevity of life, resurgence of 
infectious pathogens leading to epidemic and pandemic events has resulted in increased demands on public health professionals. This demand requires rapid identification and response to limit the dissemination of these pathogens. One of the greatest problems facing today's public health infrastructure is the greying workforce. In Illinois, for example, the average age of a State Health Agency worker was 48 years in 2007 . As a result, $~ 50 \%$ of those workers were eligible for retirement in $2012[4,5,9]$. Nationwide, the average number of public health workers eligible for retirement in 2012 was estimated to be 150000 reducing the current workforce of 500000 employees by $30 \%[4,5,9]$. As a result, laboratory workers, epidemiologists, and environmental health workers will become burdened by an increased work-load if the workforce cannot be replenished with properly trained personnel.

In 2001, the Center for Disease Control (CDC) put forth recommendations to the Appropriations Committee of the U.S. Senate outlining specific goals that needed to be attained by the year 2010 to ensure a strong public health workforce with specific emphasis placed on epidemiologists, environmental health specialists, and laboratorians [7]. Despite these suggestions, the U.S. public health workforce has continued to decline as a consequence of: i) aging and retirement of public health workers, ii) workers opting into early retirement as a result of pension reform, and iii) general lack of knowledge or interest of persons to pursue public health related employment. In addition, cutbacks, layoffs, and hiring freezes brought on by budget cuts to state and local public health departments have resulted in a smaller public health workforce struggling to serve an ever increasing population with rapidly evolving technically advanced procedures.

\section{Development of the graduate degree in Public Health Laboratory Sciences (PHLS)}

The CDC also recommended that by 2010 , each public health laboratory have access to rapid and high quality testing procedures and the utilization of standards for specimen collection, transport, testing, and reporting. ${ }^{7}$ To meet these demands, modern public health laboratories are relinquishing traditional testing strategies in favor of molecular methods which can provide definitive results in a matter of hours instead of days to weeks after receiving a clinical sample. The increasing demand for well-educated and trained professionals underscored the need to establish an advanced degree program. In August of 2005, SIU-SOM in partnership with IDPH implemented the Public Health Laboratory Sciences (PHLS) degree program to address this need. The program is designed to expose students to advanced education in the basic sciences and public health. Didactic coursework is augmented with hands-on training in the IDPH laboratories where students receive bench-top experience with clinical, environmental, and biothreat samples during routine, epidemic, and pandemic events. In addition, PHLS graduate students meet Clinical Laboratory Improvement Amendments (CLIA) certification requirements. This in-depth training combined with a wellrounded academic curriculum ensures development of highly-qualified laboratorians who are well-versed in advanced biological disciplines. The academic and laboratory training experiences of the PHLS program are detailed below and summarized in Figure 1.

\begin{tabular}{|c|c|c|c|}
\hline \multicolumn{2}{|l|}{ Fall Year 1} & \multicolumn{2}{|c|}{ Spring Year 1} \\
\hline Course & Credits & Course & Credits \\
\hline Biochemistry I & 3 & Immunology & 3 \\
\hline Introduction to Public Health & 3 & Biochemistry II & 3 \\
\hline Public Health Laboratory Disciplines & 3 & Public Health Laboratory Training & 3 \\
\hline Student Seminar & 1 & Student Seminar & 1 \\
\hline \multicolumn{2}{|c|}{10 hours per week in Public Health Laboratory } & \multicolumn{2}{|c|}{15 hours per week in Public Health Laboratory } \\
\hline \multicolumn{4}{|c|}{ Summer } \\
\hline Course & \multirow{2}{*}{$\begin{array}{c}\text { Credits } \\
6\end{array}$} & Work Hours & \\
\hline Public Health Laboratory Training & & \multicolumn{2}{|c|}{35 hours per week in Public Health Laboratory } \\
\hline \multicolumn{4}{|c|}{$\begin{array}{l}\text { Rotation 1: Carbondale Laboratory (Sexually Transmitted Disease Testing) } \\
\text { Rotation 2: Chicago Laboratory (Newborn Screening) }\end{array}$} \\
\hline \multicolumn{2}{|l|}{ Fall Year 2} & \multicolumn{2}{|c|}{ Spring Year 2} \\
\hline Course & Credits & Course & Credits \\
\hline Environmental Chemistry & 3 & Medical Microbiology & 3 \\
\hline Bacterial Genetics & 3 & Public Health Laboratory Training & 6 \\
\hline Public Health Laboratory Training & 3 & Student Seminar & 1 \\
\hline Student Seminar & 1 & & \\
\hline 25 hours per week in Public Health La & tory & 25 hours per week in Public Health & tory \\
\hline
\end{tabular}

Figure 1: The PHLS curriculum.

Schematic demonstrating the academic and laboratory training requirements to complete the non-thesis MS degree in PHLS. Total academic credits equal 46 hours (28 of didactic courses and 18 of laboratory training). Core science courses include Biochemistry I, Biochemistry II, Immunology, Bacterial Genetics and Student Seminar,and Public health program-specific courses include Introduction to the Public Health, Public Health Laboratory Disciplines, Environmental Chemistry, Clinical Microbiology and Research Methods. Hands-on training is received in the laboratory (Public Health Laboratory Training) throughout the program with rotations in the Carbondale and Chicago laboratories completed in the summer term between year 1 and year 2. 


\section{PHLS Curriculum}

This non-thesis Master's degree requires completion of 46 credit hours (28 credits of academic courses and 18 credits of laboratoryspecific training) over 24 months. Each class consists of 3 to 4 students with undergraduate degrees in the physical, biological and clinical sciences including biology, chemistry, microbiology, medical technology, or public health and others. Core science courses are identical to those taken by all Master's and $\mathrm{PhD}$ graduate students in the Microbiology, Biochemistry and Molecular Biology (MBMB) graduate program: Biochemistry I, Biochemistry II, Immunology, Bacterial Genetics and Student Seminar. The curriculum also includes program-specific courses: Introduction to the Public Health, Public Health Laboratory Disciplines, Environmental Chemistry, Clinical Microbiology and Research Methods. The majority of the coursework is completed during the first academic year. Hands-on training is completed in the public health laboratory where students are trained in diagnostic microbiology, environmental microbiology, blood lead analysis, and molecular diagnostics. By the end of the first year, students have completed 20 academic credits and have accumulated nearly 500 hours of hands-on training in the laboratory. During the summer term (May through August), students do not take academic courses and instead devote all of their time to laboratory training ( 35 hours per week). This period focuses on practical application of knowledge gained during the first year to support day-to-day laboratory work. Students also have opportunities to participate in field work with local health department staff. Activities include animal control, family case management, food sanitation, immunization clinics and senior home visits. This exposes them to the wider practices of public health. In addition, students travel to IDPH labs in
Chicago and Carbondale to rotate through testing sections specific to those locations, such as newborn screening and sexually transmitted disease testing, respectively. These opportunities increase exposure to advanced testing procedures and personnel at these locations. During the second year, students complete the remaining academic requirements totaling 11 credit hours. Students spend substantially more time in the laboratory ( 25 hours per week versus 10 to 15 in the first year) where they further refine and expand their practical skill sets. By the end of the program, students have attained training in clinical, environmental and molecular testing protocols for infectious, sexually-transmitted and heritable disease as well as food, milk, water, blood lead testing, and the identification of bio-threats as part of the Illinois Public Health Preparedness Center in collaboration with local and federal law enforcement agencies using state-of-the-art equipment (Table 1).

\section{Laboratory Training Opportunities and Objectives}

Students have the opportunity to train in all three of the IDPH laboratories based in Springfield, Carbondale, and Chicago. These labs perform differing tests to support public health epidemiology programs by providing surveillance data with the goal of improving public health and environmental quality throughout Illinois. Each laboratory participates in numerous certification programs to ensure the accuracy of testing data. All three laboratories have a CLIA certificate and Select Agent certificate to ensure quality clinical laboratory testing and biological threat agent testing, respectively. The Chicago laboratory is accredited by the American Industrial Hygiene Association Laboratory Accreditation Programs (AIHA-LAP, LLC) to test paint, soil, dust wipes, and air filters to determine the level of lead in

Table 1: Procedures and equipment used in clinical, environmental and molecular testing protocols

\begin{tabular}{|c|c|c|}
\hline Section & Testing Procedures & Equipment \\
\hline $\begin{array}{l}\text { Clinical } \\
\text { Microbiology }\end{array}$ & 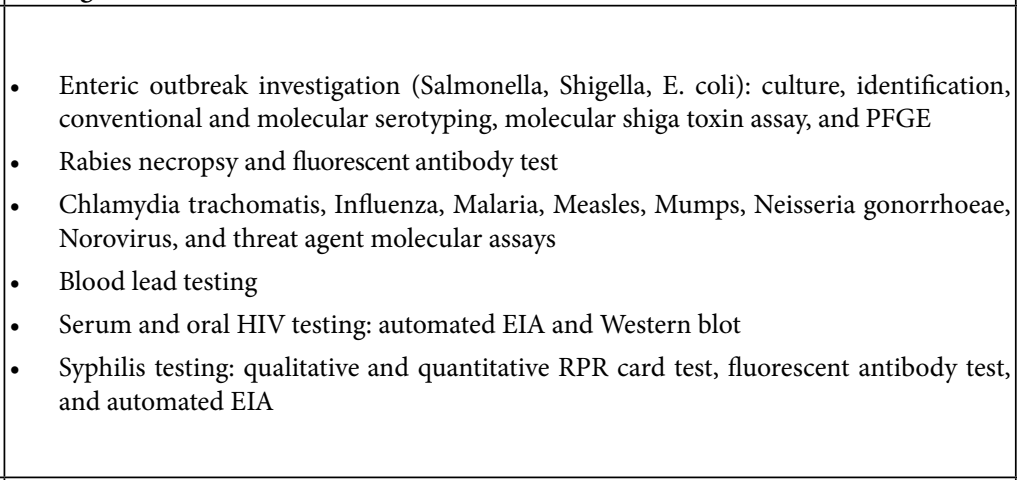 & 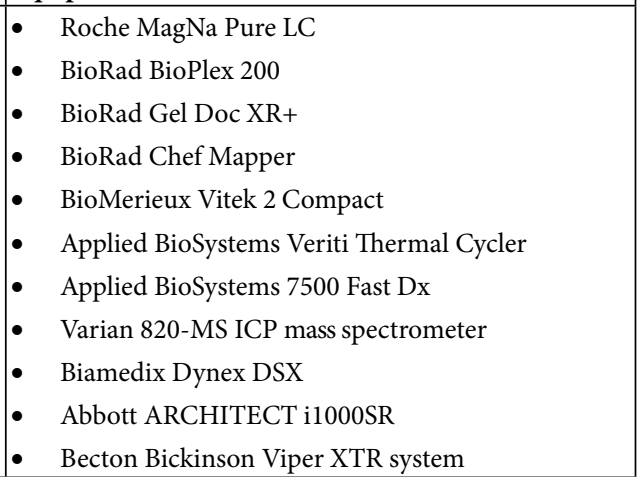 \\
\hline $\begin{array}{l}\text { Environmental } \\
\text { Microbiology }\end{array}$ & $\begin{array}{l}\text { - Determination of antibiotic residues in milk by Charm } 3 \text { SL3 test and Delvotest } 5 \text { Pack } \\
\text { - Testing of dairy products for Aerobic and Coliform spp. by Petrifilm techniques and } \\
\text { alkaline phosphatase by Charm PasLite test } \\
\text { - Detection of Coliforms and E. coli in water by Colilert and Quanti-Tray procedures }\end{array}$ & $\begin{array}{ll}\text { - } & \text { BioMerieux VIDAS } \\
\text { - } & \text { BioMerieux VITEK } \\
\text { - } & \text { CHARM Novalum Rosa Reader }\end{array}$ \\
\hline Other Programs & $\begin{array}{l}\text { - IDPH Laboratory Improvement Section (LIS), where CLIA, environmental and State } \\
\text { discussed. } \\
\text { - Milk split proficiency program: preparation of unknown samples to be testedby milk la } \\
\text { - IDPH Laboratory Division Chief: discussed laboratory managementand budgeting isst } \\
\text { IDPH Select Agent Responsible Official (RO): discussed regulations governing the Select } \\
\text { security risk assessment approved to work with threat agents. RO also discussed Laboratory }\end{array}$ & $\begin{array}{l}\text { of Illinois regulations governing laboratory testing was } \\
\text { aboratories } \\
\text { ues } \\
\text { Agent Program, CDC inspections, and was Select Agent } \\
\text { y Response Network (LRN) threat agent testing. }\end{array}$ \\
\hline
\end{tabular}

Abbreviations: PFGE, pulsed-field gel electrophoresis; HIV, human immunodeficiency virus, EIA, Enzyme immunoassay; RPR, rapid plasma reagin; IDPH, Illinois Department of Public Health; CLIA, clinical laboratory improvement amendments 
these samples. The Carbondale and Chicago laboratories are certified water microbiology and dairy labs. The Springfield laboratory is accredited by the U.S. Food and Drug Administration (FDA) and U.S. Environmental Protection Agency (EPA) for dairy and drinking water testing, respectively. As a result, students have an opportunity to gain broader knowledge in all medical and scientific disciplines by working with personnel who strive to ensure advanced laboratory capabilities at all three locations. The majority of the tenure is spent at the Springfield location. The IDPH Division of Laboratories in Springfield is composed of three primary sections: environmental microbiology, diagnostics/clinical microbiology, and blood lead screening. Students gain experience in laboratory testing procedures and become proficient using procedures and equipment which transcend many fields after graduation. In the environmental microbiology section, students learn procedures for water and dairy testing. Water testing is performed for nitrate and nitrite detection, fluoride detection, and $\mathrm{pH}$ determination. Dairy testing is performed for laboratory grade testing and for detection of elevated coliform counts. Students also have the opportunity to participate in the FDA milk splits proficiency testing program in accordance to the requirements of the Grade A Pasteurized Milk Ordinance. By successfully completing the proficiency test, students become certified milk analysts. In the diagnostics/clinical microbiology section, students become proficient in quantitative polymerase chain reaction (qPCR) and pulsed-field gel electrophoresis (PFGE). These methods are used to detect and characterize infections caused by typical enteric bacteria such as Salmonella, Shigella, and Escherichia coli. The experience gained with qPCR techniques extends to the detection of Mumps, Measles, Norovirus, and Influenza. Direct Fluorescent Antibody (DFA) testing procedures are learned for detection of Rabies virus. In the blood lead section, students learn to process samples and utilize procedures and equipment, such as inductively coupled plasma mass spectrometry (ICP-MS), to test for elevated lead levels in venous blood samples. In addition to these advanced techniques and skills, students learn and are expected to follow quality assurance and quality control procedures for record keeping, equipment maintenance, experimental and environmental controls in each laboratory section. Once appropriately trained, PHLS students have been utilized to enhance the effectiveness of the laboratories in performing public health services. The advanced biological, biochemical, and molecular knowledge of these students can be called upon to troubleshoot, identify, and establish new testing capabilities within IDPH laboratories in support of enhanced measures to identify infectious pathogens. For example, in 2009 the IDPH laboratory in Carbondale, IL used the molecular expertise of a PHLS graduate student to develop a biosafety level (BSL)2/BSL3 molecular testing facility for the identification of pandemic Influenza, Norovirus, HIV, Shiga-toxin (STX)-producing microbial pathogens, and bio-terror select agents.

\section{Comparison of the PHLS degree to traditional MPH programs}

To illustrate the novelty of the PHLS program, we compared our graduate curriculum against the average of five well-established and accredited MPH graduate programs. This comparison was performed by dividing the core curriculum into three categories: i) biological science courses (Fig. 2A, B; blue), ii) didactic public health associated courses (Fig. 2A, B; grey), and iii) laboratory training and application and/or equivalent internship experience (Fig. 2A, B; orange). The model was applied to determine the relative emphasis (percentage) that each program dedicated to establishing an academic curriculum which would best meet the demands necessary to increase laboratory scientists in the public health workforce. Despite the presence of all three core components in both the PHLS and MPH programs, the distributions of those components vary widely. For example, the primary focus of MPH academic programs is on public health administration and policy with courses such as: Justice and Resource Allocation, and Ethical Basis of the Practice of Public Health making up $68 \%$ of the curriculum (Fig. 2A) [10]. The remaining academic instruction covers the biological sciences (28\%). Practical experience acquired in the laboratory or through an internship makes up less than $5 \%$ of MPH degree requirements (Fig. 2A). In contrast, the PHLS program provides a more even distribution among public health policy, biological sciences, and laboratory experience (Fig. 2B). Thus, PHLS graduates are well-versed in public health policy, trained

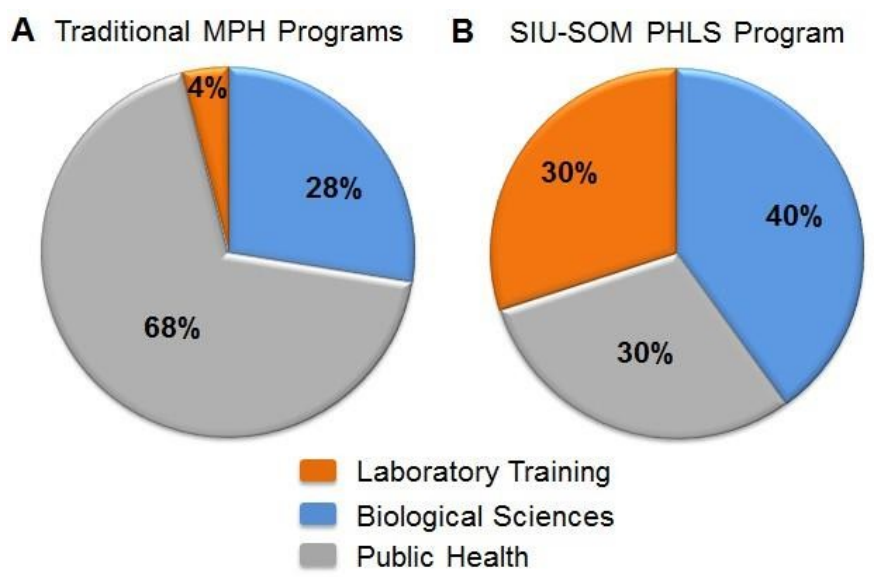

Figure 2: Comparison of academic curriculum for a traditional MPH versus the PHLS program.

Core didactic courses in public health (grey), biological sciences (blue), and laboratory training or equivalent internship experience (orange) were identified within (A) the average of five well-established and accredited MPH programs, and compared against the same criteria for the (B) SIU-SOM PHLS program. Each colored section was defined as the cumulative percentage (\%) of courses offered within each respective area as described in the course description from each institution [10-12]. 
to perform clinical and environmental testing, and knowledgeable of core biological sciences used to design, implement, an troubleshoot diagnostic assays utilized by modern public health facilities.

\section{Current success of the PHLS program}

Despite the vacancies of available public health professionals, recent reports have indicated that there remains a lack of enthusiasm and interest in public health careers [4]. This may be due, in part, to a lack of available nationwide public health programs designed to motivate and instruct public health professionals to pursue careers in modern public health facilities. This may be an issue for some traditional MPH programs. MPH programs frequently lack clear direction for graduate students other than to encourage the graduates to pursue a position as a public health department administrator, public health program manager, or similarly titled position. This problem is further compounded by the fact that there is a nebulous boundary which separates public health occupations from similar health care occupations. However, public health disciplines are very focused on community health and population health at the local and national level, whereas health care occupations focus on the health of the individual. The PHLS program is designed to train graduate students in the varied aspects of public health policies and procedures while emphasizing and focusing on the accrual of in-depth scientific knowledge and laboratory training to be able to effectively analyze and diagnose threats to the health of the community/ population. To date, 22 students have completed the PHLS program. Over $50 \%$ of these students (14 of 22) were born and raised in Illinois with the remainder coming from the continental US or abroad. The majority of students have been females (18 of 22). The overall success of any program can be measured in terms of occupational placement of students within the respective program following graduation. By these criteria, we evaluated the percentage of students that successfully completed the PHLS program by the occupational career fields in which they are currently employed. Fifteen (68\%) are currently employed in clinical laboratory scientist positions in public health occupations, six (27\%) have completed or are pursuing doctoral level education within public health related fields, and one (5\%) is employed by a biotechnology company (Fig. 3). Thus, $100 \%$ of all PHLS graduates continue to use the knowledge and technical skills obtained through the PHLS program in their chosen careers.

\section{Conclusions}

The demand for well-trained and competent public health professionals will continue to increase to meet the demand of the ever-increasing U.S population and sophistication of public health laboratory testing methods. In collaboration with IDPH, the SIUSOM has instituted a curriculum in Public Health Laboratory Sciences designed to instruct graduate students in public health and policy and the biological sciences as well as technical laboratory training. We believe this combination of education and technical experience is essential to produce public health professionals who are competent to conduct clinical testing, train personnel, and manage core public health facilities. We have found this program to be feasible utilizing existing resources and funding. In addition, the majority of core didactic course for the PHLS students are taken in conjunction with other MS and $\mathrm{PhD}$ graduate students in the MBMB program. This arrangement facilitates accrual of advanced analytical thinking skills and exposes PHLS students to unique ways of approaching problems. The benefits to the host laboratory are immediate. By the end of year 1 , students are exposed to the clinical and environmental analyses performed in the public health laboratory. This is amazingly practical, as the scientific knowledge gleaned from the concurrent science courses is immediately integrated into the laboratory analyses that the students are required to experience "at the bench". The contribution to the public health laboratory infrastructure is practically immediate, as the staff of the host laboratory orient and train the students to aid in daily conduct of tests. This has very practical utilization as the frequent spikes in test volumes (e.g. foodborne outbreak investigation, suspect bioterrorism samples, etc.) allow the staff to utilize the students-intraining to augment the laboratory's capacity. Of perhaps far greater significance, this unique "labor pool" can be accessed without resorting to the very convoluted and extended timeframe hiring process this is characteristic of most government public servant positions. We believe this program is an important and unique resource to meet public health workforce shortages. Furthermore, advances in the complexity of analyses and expanding regulatory requirements dictate that every discipline practiced in public health will demand a greater in-depth knowledge of that discipline at the outset. The end result is the development of a highly educated and experienced individual who can immediately assume lead worker duties in a modern public health laboratory, with no or very little additional laboratory training.

\section{Implications for Policy \& Practice}

- The PHLS program addresses many of the challenges identified by the Public Health Workforce Interests and Needs Survey (PH WINS).

- The PHLS program provides a well-balanced curriculum in public health and policy and the biological sciences as well as technical laboratory training.

- PHLS students contribute to the public health laboratory infrastructure.

- The host public health laboratory receives immediate benefit as scientific knowledge gained from science courses is immediately integrated into the laboratory analyses that the students are required to experience "at the bench".

- Creates a unique "labor pool" which can be accessed immediately during outbreaks or spikes in test volumes.

\section{References}

1. Institute of Medicine (2003) Who Will Keep the Public Healthy? Educating Public Health Professionals for the 21st Century.

2. State Public Health Employee Worker Shortage Report: A Civil Service Recruitment and Retention Crisis. Association of State and Territorial Health Officials. Published 2004. Accessed 31 March 2017.

3. Draper DA, Hurley RE, Lauer JR (2008) Public health workforce shortages imperil nation's health. Res Brief 4: 1-8. [crossref]

4. Master of Public Health Program - Curriculum Guide (2012) In: Health HSoP. Boston.

5. Master of Public Health Program - Curriculum Guide (2015) In: Health JHBSoP. Baltimore. 
6. Morrissey T (2006) The Affordable Care Act's Public Health Workforce Provisions: Opportunities and Challenges. Washington DC.

7. Perlino C (2006) The Public Health Workforce Shortage: Left Unchecked, Will We Be Protected? Washington, DC.

8. Public Health Infrastructure. Centers for Disease Control and Prevention. https://www.cdc.gov/media/pressrel/fs020514.htm. Published 2001. Accessed March 30, 2017.

9. Rosenstock L, Silver GB, Helsing K, Evashwick C, Katz R et al. (2008) On Linkages: Confronting the Public Health Workforce Crisis: ASPH Statement on the Public Health Workforce. Public Health Reports 123: 395-398. [crossref]
10. Willard R. Local Health Department Job Losses and Program Cuts. National Association of County and City Health Officials. http://archived. naccho.org/topics/infrastructure/lhdbudget/upload/Job-Losses-and-ProgramCuts-5-10.pdf. Published May 2010. Accessed March 31, 2017.

11. Toossi M (.2013) Labor force projections to 2022: the labor force participation rate continues to fall. Monthly Labor Review.

12. 2-Year Master of Public Health (MPH) in Environmental Health Sciences (2015) In: University of California BSoPH. Baltimore. 